\title{
Article
}

\section{Investigating the Characteristics of Vulnerable Referrals Made to a Multi- Agency Safeguarding Hub}

Shorrock, Sarah, Mcmanus, Michelle Ann and Kirby, Stuart Available at http://clok.uclan.ac.uk/25980/

Shorrock, Sarah, Mcmanus, Michelle Ann ORCID: 0000-0002-0095-1071 and Kirby, Stuart ORCID: 0000-0002-3049-1248 (2019) Investigating the Characteristics of Vulnerable Referrals Made to a Multi-Agency Safeguarding Hub. Policing: A Journal of Policy and Practice, 13 (2). pp. 201-212. ISSN 17524512

It is advisable to refer to the publisher's version if you intend to cite from the work. http://dx.doi.org/10.1093/police/paz003

For more information about UCLan's research in this area go to http://www.uclan.ac.uk/researchgroups/ and search for <name of research Group>.

For information about Research generally at UCLan please go to http://www.uclan.ac.uk/research/

All outputs in CLoK are protected by Intellectual Property Rights law, including Copyright law. Copyright, IPR and Moral Rights for the works on this site are retained by the individual authors and/or other copyright owners. Terms and conditions for use of this material are defined in the policies page.

\section{CLoK}

Central Lancashire online Knowledge www.clok.uclan.ac.uk

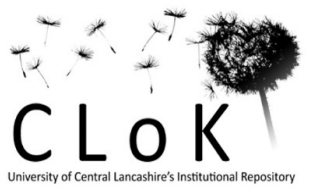


RUNNING HEAD: Investigating the Characteristics of Vulnerable Referrals made to a MultiAgency Safeguarding Hub (MASH).

\section{ABSTRACT:}

Multi-Agency Safeguarding Hubs (MASH) have been a feature of safeguarding processes since 2010, aiming to increase information sharing, joint decision-making and co-ordinated interventions between safeguarding agencies. However, understanding surrounding the mechanisms underpinning MASH, and who they protect, is limited. This paper attempts to bridge this gap in knowledge by quantitatively examining referrals made to one MASH location in the North of England between $1^{\text {st }}$ October 2013 and $30^{\text {th }}$ November 2014 $(n=51,264)$. The findings outline general features of a MASH framework, whilst demonstrating that demand placed upon MASH is influenced by a range of static and dynamic risk factors, including gender, age and ethnicity. The study highlights the complex nature of referrals made to MASH and suggests that whilst MASH has taken a step towards a multi-agency approach to safeguarding, questions regarding MASHs ability to effectively safeguard vulnerable individuals at the earliest opportunity remain. 
RUNNING HEAD: Investigating the Characteristics of Vulnerable Referrals made to a MultiAgency Safeguarding Hub (MASH).

Investigating the Characteristics of Vulnerable Referrals made to a Multi-Agency Safeguarding Hub (MASH).

\section{Introduction}

Unlike previous safeguarding mechanisms, which have typically involved single agency decision-making processes, Multi-Agency Safeguarding Hubs (MASH) aim to identify and manage risk at the earliest opportunity by promoting a collaborative approach to safeguarding. However, who is referred to MASH and for what reasons, is often overlooked within the literature, making it difficult to establish whether MASH is effectively identifying and managing those most at risk of harm or abuse. This study, therefore, explores the characteristics of cases referred to MASH and the mechanisms that have been introduced to protect society's most vulnerable individuals.

\section{Literature Review}

Multi-agency partnerships have been present within England since the 1980s. The Children Act 1989 established one of the first statutory requirements for inter-agency collaboration and joint working, concerning children and young people. Since then, other acts, policies and guidelines have been introduced to promote a multi-agency approach to safeguarding. These have included: recommendations emerging from serious case reviews, particularly Lord Laming's 2003 report into the death of Victoria Climbie; the Equality Act 2010; and the regular publication of Working Together to Safeguard Children. Throughout this period, the importance of providing a multi-agency approach to safeguarding vulnerable individuals has been well documented. 
RUNNING HEAD: Investigating the Characteristics of Vulnerable Referrals made to a MultiAgency Safeguarding Hub (MASH).

The introduction of MASH, which is accredited to Nigel Boulton (Golden et al., 2011), is a recent example of multi-agency practitioners taking a shared responsibility to identify and manage vulnerability at the earliest opportunity. The rationale for MASH emerged from Boulton's experience of being a police officer, where he recognised that relevant intelligence needed to be collected first, before effective safeguarding decisions could be made. This observation has been reflected in many serious case reviews, illustrating that a lack of information sharing between agencies can result in vulnerable individuals being exposed to unnecessary harm or abuse (Laming, 2003; Haringey, 2009). Thus, by co-locating safeguarding agencies, MASH aims to move towards a more collaborative approach, increasing the likelihood of safeguarding decisions being holistic, effective and more proactive (Cullinan, 2013; Hanson et al., 2015). Agencies that are co-located in MASH include (but are not limited to), the Police, Children Services, Adult Services and Health Services (Norfolk Adult Safeguarding Board, 2014).

Since 2010, most local authorities have embedded a MASH framework into their safeguarding practices, allowing the gaps within traditional silo approaches to be acknowledged and rectified. Whilst a legal definition of MASH does not exist, the Home Office (2014) recognises that most MASH frameworks are based upon three core elements; (1) information sharing, (2) joint decision-making and (3) co-ordinated interventions. Across England and Wales, MASH frameworks vary, with MASH having a primary role of providing a 'proportionate, timely and coordinated approach' to safeguarding (Allen and Wilde, 2013: 5). For most MASH frameworks, safeguarding vulnerable children is the main priority, whilst others also focus upon the identification and management of vulnerable adults and domestic abuse victims. Therefore, MASH is a multi-faceted safeguarding initiative, which 
RUNNING HEAD: Investigating the Characteristics of Vulnerable Referrals made to a MultiAgency Safeguarding Hub (MASH).

aims to protect society's most vulnerable individuals, by establishing a secure environment in which practitioners can confidentially share information and make joint decisions regarding safeguarding interventions.

However, since the introduction of MASH and the co-location of agencies, a lack of information sharing amongst safeguarding practitioners has been reported. Reasons why agencies fail to share important information varies, but often includes: concerns over confidentiality; the rules outlined in Article 8 of the Human Right Act 1998 and The Data Protection Act 1998. Within the safeguarding literature, it is widely accepted that these laws do not prevent the sharing of information, but act as guidelines to ensure the sharing of sensitive information is proportionate and necessary (Boulton, 2013; London Safeguarding Children Board, 2013). Subsequently, MASH have been encouraged to view the non-sharing of information to be more damaging than sharing information (HM Government, 2015), with professionals often having the authority to share information even if consent has not been obtained. Such authority may come from Section 115 of the Crime and Disorder Act 1998 or may be implied from Sections 10 and 11 of the Children Act 1984.

Most MASHs comply with the Caldicott Principles (Caldicott, 1997), which provide a set of standardised guidelines, ensuring the sharing of confidential information is justifiable. Hence, decisions made within MASH can be based upon a common set of regulations, helping to reduce anxieties around what information should be shared and when. Colocation also encourages the sharing of confidential information, since practitioners operate in a 'fire walled environment' (Nib Consulting, 2014), with shared information only being used to inform safeguarding decisions. This enables joint decision-making to become routine practice, whilst enhancing the likelihood of interventions being proportionate, 
RUNNING HEAD: Investigating the Characteristics of Vulnerable Referrals made to a MultiAgency Safeguarding Hub (MASH).

necessary and effective. To achieve such objectivity, most MASHs utilise a three-stage approach when deciding upon the most appropriate course of action, with these stages incorporating both risk management and joint decision-making techniques. Figure 1 provides an example from a MASH location in the North of England.

\section{[FIGURE 1 HERE]}

The first stage of this process is the 'input stage', whereby agencies or practitioners raise their concerns. Cases that meet the MASH criteria then advance to the 'processing stage'. During this stage, the agency that raised the initial concern conducts a risk assessment to establish the urgency of the case and a timeframe in which interventions need to be implemented. This stage also provides agencies with an opportunity to gather, collate and analyse relevant information, with the completion of this stage leading to the 'decision-making stage'. At this stage, MASH professionals review the case and record a decision about next steps, with an option to record a decision of 'No Further Action' being removed from the MASH process (Gloucestershire, 2014). At a minimum, each MASH referral should be passed on to the relevant agency for further monitoring or investigation, ensuring all concerns are adequately reported, recorded, reviewed and acted upon. Equally, MASH requires safeguarding agencies to co-ordinate their interventions to increase the likelihood of vulnerable individuals being placed on the most 'appropriate service pathway, at the earliest opportunity' (Lancashire CYP Trust Board and LSCB, 2012:1). This reduces the likelihood of an individual being repeatedly harmed or abused (Crockett et al., 2013), whilst allowing resources to be directed towards the riskiest situations. 
RUNNING HEAD: Investigating the Characteristics of Vulnerable Referrals made to a MultiAgency Safeguarding Hub (MASH).

By reviewing the core components of $\mathrm{MASH}$, it can be argued that the establishment of a multi-agency approach to risk management has addressed (in theory), the weaknesses of previous safeguarding mechanisms. Commissioned reports support this assumption (Golden et al., 2011; Allan and Wilde, 2013; Crockett et al., 2013), highlighting how MASH has: increased collaboration between safeguarding agencies; reduced the number of unnecessary referrals; assisted agencies to identify gaps in services earlier; and enabled responses to be more effective and efficient. Whilst these reports provide an overview of the impact MASH has had upon safeguarding practices, they generally fail to describe who is referred to MASH and the reasons why. This study therefore, aims to address this oversight by investigating the characteristics of referrals made to one MASH location in the North of England.

\section{METHODOLOGY.}

\section{Data Source.}

To achieve the aims of this paper, data relating to a MASH location in the North West of England was provided from police systems. Agencies embedded within this MASH site included the police, child social services, adult services, probation, youth offending teams, health, mental health, education, children centres, fire and rescue and independent domestic violence advisors (IDVA). Across these agencies, a total of 108 practitioners contributed to the MASH process, with 35\% of MASH practitioners coming from child social services $(n=38)$. The police accounted for $31 \%$ of MASH practitioners $(n=34)$, whilst $13 \%$ of practitioners came from adult services $(n=14)$. Health represented $8 \%$ of practitioners $(n=9)$, with all other agencies contributing between one and four practitioners ( $n=12,11 \%$ ). 
RUNNING HEAD: Investigating the Characteristics of Vulnerable Referrals made to a MultiAgency Safeguarding Hub (MASH).

This MASH site only processed referrals made by the police, with a future intention of processing referrals from other agencies. Between the $1^{\text {st }}$ October 2013 and $30^{\text {th }}$ November 2014, 51,264 referrals were made. These records were generated by a police officer attending an incident and identifying at least one individual to be 'vulnerable'. In these referrals, demographic information relating to the victim (Lead Referral: LR) and the individual perceived to have caused harm (Suspect) is recorded, alongside the characteristics of the situation and a brief description as to why the LR was perceived to be vulnerable. The term 'vulnerable' is used to describe individuals most at risk of being exposed to risky situations, with vulnerability definitions and thresholds reflecting those used by the police force in the chosen MASH location. Similarly, 'safeguarding' refers to the responsibility of MASH agencies to protect vulnerable individuals from being harmed or abused, with such responsibilities being defined by organisational practices.

A referral is also associated with an event description of either Vulnerable Children (aged 17 or younger), Vulnerable Adults or Domestic Abuse. Domestic Abuse cases reflect the Government's (2016) definition and relate to an individual over the age of 16, who has been the victim of violence or abuse by an intimate partner or family member. If it involves a vulnerable child or adult, it is referred as a Domestic Abuse case, with other potential vulnerabilities being noted. Therefore, all cases within the analysis are unique and not included in more than one event description.

Based upon this assessment, it is the responsibility of the referring officer, or staff member, to assign the case a risk level (high, medium or standard), using police guidelines. If there is evidence that an individual is, or has been subjected to significant harm, the case is recorded as high-risk and should be processed within 4-hours. A medium-risk case is 
RUNNING HEAD: Investigating the Characteristics of Vulnerable Referrals made to a MultiAgency Safeguarding Hub (MASH).

defined as having the potential for an individual to suffer significant harm, with such cases assigned a 24-hour processing timeframe. If there are concerns surrounding an individual's health or well-being, a standard-risk level is assigned to the case, alongside a 72-hour processing time. Once all this information has been recorded, a referral to MASH is made and the process commences. This study analysed the information from referral forms, albeit there was missing data.

On a MASH referral form, the gender of a LR and Suspect is reported as either female, male or unknown. The age of a LR and Suspect had to be calculated, since a referral form only specified an individual's date of birth. Age therefore, had to be calculated by subtracting the 'Recorded Date' (see below for definition) away from an individual's date of birth, resulting in a range of ages emerging. When reporting the ethnicity of a LR and Suspect a referral form has seven standardised options: Asian, Black, Chinese, Middle Eastern, White Northern European, White Southern European, or Unknown. For this study, ethnicity was collapsed into four groups Asian, Black, White (White Northern and Southern European), Other (Chinese and Middle Eastern) and Unknown.

To assist with identifying the relationship between the Suspect and LR, a MASH referral form presents twelve relationship statuses: Aunt/Uncle, Brother/Sister, Cousin, Grandparent, Niece/Nephew, Parent, Partner/Ex, Position of Trust, Son/Daughter, Stranger, Other and Unknown. The reason to why a referral is being made is also recorded on a referral form, with the referring officer having the ability to select one or more relevant referral reason. These referral reasons include: Channel (terrorism) Child Sexual Exploitation (CSE), Death, Domestic Violence, Financial Abuse, Forced Marriage, Honour Based Abuse (HBA), Missing from Home (MFH), Neglect, Physical Abuse, Sexual Abuse and 
RUNNING HEAD: Investigating the Characteristics of Vulnerable Referrals made to a MultiAgency Safeguarding Hub (MASH).

Trafficking/Slavery. To indicate whether one or more of these referral reasons was applicable to a case, the referral form logged a ' $\mathrm{Y}$ ' for ' $y e$ ' $^{\prime}$ and a ' $N$ ' for 'no'. Within this study, referral reasons of Death, Financial Abuse and Trafficking/Slavery were grouped together as 'Other', due to less than $1 \%$ of cases $(n=198)$ reporting these as referral reasons.

Finally, a MASH referral form records three key dates (see Figure 2). First, the date the initial incident occurred, with this being known as the 'Recorded Date'. Second, the 'Referral Date', which relates to the date the case was referred to MASH. Finally, the 'Finalisation Date', which represents the date the case was closed by MASH. To calculate the Referral Time, the Recorded Date was subtracted from the Referral Date. To identify the time taken for a case to be processed, the Referral Date was subtracted from the Finalisation Date. This study analysed the information recorded on referral forms, with Unknown cases being omitted from the analysis.

\section{[FIGURE 2 HERE]}

Data Analysis.

Inferential statistics were conducted using SPSS, enabling relationships between the different variables to be identified. All data was normally distributed, allowing parametric tests to be carried out. Chi-square tests were used to explore categorical data, whilst interval data, with two levels to the independent variable, were analysed using independent sample t-tests. For interval data, which involved the comparison of three or more levels to the independent variable, one-way analysis of variance (ANOVA) tests were used. 
RUNNING HEAD: Investigating the Characteristics of Vulnerable Referrals made to a MultiAgency Safeguarding Hub (MASH).

\section{FINDINGS.}

\section{General Findings.}

Between $1^{\text {st }}$ November 2013 and $30^{\text {th }}$ November 2014, 51,264 LR were referred to MASH (Table 1), with $59 \%$ of these cases relating to Domestic Abuse (DA) incidents ( $n=30,231)$. Vulnerable Child (VC) referrals accounted for $21 \%$ of cases $(n=10,691)$, whilst $20 \%$ of referrals were recorded as a Vulnerable Adult (VA) incident ( $n=10,342)$. Across the event descriptions, $3 \%$ of referrals involved more than one Suspect $(n=1,581)$, resulting in 52,721 Suspects being included in the analysis.

A Chi-Square analysis found a significant difference between event description and risk level, $\left(X^{2}(4)=5971.79, p<.001\right.$, Cramer's $\left.V=.241\right)$. VC cases were more likely to be referred as a medium-risk ( $n=5,083,48 \%)$, whereas VA $(n=4,521,44 \%)$ and DA $(n=18,067$, $60 \%)$ referrals were predominantly associated with standard-risk referrals.

For all event descriptions, Lead Referrals (LR) were predominantly female ( $n=33,569$, $68 \%)$, whilst Suspects were mainly male ( $n=28,630,78 \%)$. The relationship between event description and gender was found to be significant for both $\operatorname{LR}\left(X^{2}(2)=2942.436, p<.001\right.$, Cramer's $V=.244)$ and Suspects $\left(X^{2}(2)=788.875, p<.001\right.$, Cramer's $\left.V=.147\right)$. For instance, $77 \%$ of DA LR were female ( $n=22,762)$, compared to $81 \%$ of Suspects being male $(n=23,918)$.

The age of $\operatorname{LR}$ was also found to be significant $(F(2,51261)=20099.222, p<.001)$, with VA LR $(M=49, S D=21.87)$ being significantly older than VC $(M=10, S D=6.32)$ and $D A$ $(M=35, S D=12.79) L R$. Equally, the analysis revealed a significant difference between the age of Suspects and the event description assigned to a referral $(F(2,37242)=138.648 p<.001)$. VA Suspects $(M=38, S D=16.25)$ were found to be significantly older than $V C(M=33, S D=12.7)$ 
RUNNING HEAD: Investigating the Characteristics of Vulnerable Referrals made to a MultiAgency Safeguarding Hub (MASH).

and DA ( $M=34, S D=11.81)$ Suspects, whilst DA Suspects were significantly older than VC Suspects.

The ethnicity of a LR was reported in $97 \%$ of cases $(n=49,564)$ and for $69 \%$ of Suspects $(n=36,367)$. A higher proportion of LR $(n=46,053,93 \%)$ and Suspects $(n=33,032$, 91\%) came from a White background, with individuals from an Asian ethnicity accounting for $6 \%$ of $\operatorname{LR}(n=2,884)$ and $7 \%$ of Suspects $(n=2,668)$. This trend was replicated across all event descriptions, with a significant interaction between LR ethnicity and event description being found $\left(X^{2}(6)=33.187, p<.001\right.$, Cramer's $\left.V=.118\right)$.

Multiple Chi-square tests evidenced a significant association between event description and the reason a referral was made to MASH ( $p s<.001)$. VC referrals were most likely to identify Sexual Abuse as a referral reason $(n=2,106,20 \%)$, whilst VA cases typically recorded a referral reason of Physical Abuse ( $n=1,253,12 \%)$. Domestic Abuse cases used this as a referral reason $(n=30,231,100 \%)$, with Physical Abuse being cited on $7 \%$ of cases $(n=1,977)$. Across all event descriptions, $61 \%$ of cases identified Domestic Abuse as a referral reason $(n=31,160)$.

A significant interaction between event description and relationship status was found, $\left(X^{2}(22)=23617.52, p<.001\right.$, Cramer's $\left.V=.571\right)$. Of those that reported a relationship status ( $n=36,276,71 \%)$, Partner or Ex-Partner was the most commonly cited $(n=24,338$, 67\%). Of the VC cases that reported the relationship between LR and Suspect $(n=4,469$, $42 \%), 50 \%$ recorded a relationship of Parent $(n=2,230)$. For VA cases, only $17 \%$ of referrals cited a relationship status $(n=1,762)$, with $39 \%$ of these cases identifying a relationship of Other ( $n=686)$. Finally, 98\% of DA cases recorded the relationship between LR and Suspects 
RUNNING HEAD: Investigating the Characteristics of Vulnerable Referrals made to a MultiAgency Safeguarding Hub (MASH).

( $n=30,018$ ), with $79 \%$ of referrals relating to incidents between Partners or Ex-Partners $(n=23,650)$. Table 1 provides a summary of key findings.

\section{[TABLE 1 HERE]}

\section{Timeframes.}

This section of the results focuses upon the referral and processing time of cases by event description and risk level. By analysing these timeframes, the notion of MASH requiring all referrals to be processed with 72-hours can be investigated, alongside whether the event description associated with a referral influenced referral and processing times. Due to this focus, differences in referral and processing times by LR and Suspect demographic characteristics was not deemed relevant, therefore omitted from the analysis.

The time taken for a referral to be made to MASH significantly differed by event description, $(F(2,51261)=78.669, p<.001)$. DA cases were referred much quicker $(M=1$ day, $S D=19.16)$ than VC ( $M=6$ days, $S D=64.27)$ and $V A(M=6$ days, $S D=59.36)$ referrals (Table 2$)$. The average referral time across all event descriptions was 3 days (SD=41.77). Processing times also significantly differed across the event descriptions, $(F(2,51261)=100.102, p<$ $.001)$. For all cases, the mean processing time was 10 days $(S D=42.64)$, with $D A$ referrals being processed significantly quicker than VC ( $M=14$ days, $S D=64.73)$ and VA $(M=13$ days, $\mathrm{SD}=59.70)$ cases.

When timeframes were analysed by risk level, a significant difference in referral time was identified, $(F(2,51261)=22.47, p<.001)$. Specifically, medium ( $M=3$ days, $S D=34.11)$ and standard ( $M=3$ days, $S D=33.16)$ risk cases were referred significantly quicker than highrisk cases ( $M=6$ days, $S D=73.60)$. A significant difference in processing time was also found 
RUNNING HEAD: Investigating the Characteristics of Vulnerable Referrals made to a MultiAgency Safeguarding Hub (MASH).

$(F(2,51261)=50.30, p<.001)$, with high $(\mathrm{M}=10$ days, $\mathrm{SD}=73.96)$ and standard $(\mathrm{M}=8$ days, $\mathrm{SD}=33.75)$ risk cases being processed significantly quicker than medium-risk cases $(\mathrm{M}=13$ days, $\mathrm{SD}=34.94)$.

[TABLE 2 HERE]

\section{DISCUSSION.}

Research around Multi-Agency Safeguarding Hubs (MASH) has previously failed to explore who is referred to MASH and for what reasons. To bridge this gap, this paper focused upon 51,264 referrals, made to one MASH location in a 13-month period. To fully understand the characteristics of these referrals, the data was analysed based upon event description. Through this process, trends associated with individuals referred to MASH, alongside the demand placed upon MASH emerged, allowing the effectiveness and complexity of the MASH framework to be better understood.

Most referrals processed by the chosen MASH site related to domestic abuse (DA), reflecting the notion that DA is a prevalent form of abuse within today's society (Office for National Statistics, 2017). Thus, whilst MASH may have been established to address vulnerability in childhood, practices and processes have been transferred to help identify and manage DA at the earliest opportunity, as well as other vulnerabilities that may occur in adulthood.

Across all event descriptions, a higher proportion of Lead Referrals (LR) were female, reinforcing the notion that women are more susceptible to becoming the victims of abuse (Office for National Statistics, 2016; Stevens et al., 2016). Consistent with previous studies, Suspects were predominantly male and more likely to expose individuals to vulnerable 
RUNNING HEAD: Investigating the Characteristics of Vulnerable Referrals made to a MultiAgency Safeguarding Hub (MASH).

situations (Modleski, 2015; Finkelhor et al., 2014). A White ethnicity was also recorded for the majority of both LR and Suspects, implying that offenders and victims often come from similar ethnic backgrounds.

The mean age of VC LR was found to be 10 years old, with this finding reflecting previous trends which imply that children aged 10 or older are at a heightened risk of being harmed or abused (Department of Education, 2015; Radford et al., 2011). Explanations as to why children aged 10 or older are at an increased risk of being exposed to vulnerable situations are limited. One reason may relate to young children not perceiving themselves to be vulnerable (Cooper, 2018), causing a child to be exposed to risky situations until concerns are raised and acted upon. The onset of puberty, alongside an increased risk of children aged 7 to 11 being sexually abused (Hassan et al., 2015; Runyan et al., 2002), may also explain why children aged 10 are more likely to be referred to safeguarding agencies. Engagement in risk taking behaviour may be another explanation, with research illustrating that as children get older, they start to push boundaries and seek out activities that are novel, irrespective of the consequences (Braams et al., 2015; Gullone and Moore, 2000). For instance, Ofcom (2017) has found that one in four children aged 8 to 11 has a social media profile, with access to the internet increasing the likelihood of a child sharing personal information or images with a stranger (Livingstone and Palmer, 2012). Thus, whilst children are intrinsically vulnerable due to their age (Furedi, 2013), factors associated with becoming a teenager might rationalise why children aged 10 are most at risk of being referred to MASH.

The average age of VA LR was 49 years, contradicting the assumption that adults aged 65 and older are most at risk of being referred to safeguarding boards (HSCIC, 2015; 
RUNNING HEAD: Investigating the Characteristics of Vulnerable Referrals made to a MultiAgency Safeguarding Hub (MASH).

Trainor and Penhale, 2015). The age of DA victims also conflicted with previous research, with a higher proportion of DA referrals involving victims aged 30 to 40 , contradicting the notion that those aged 20 to 30 years are at a heightened risk of becoming domestic abuse victims (Abramsky et al., 2011; SafeLives, 2015). These findings therefore, infer that the likelihood of being exposed to vulnerable situations is associated with an individual's age, with children and adults aged 30 to 50 at an increased risk of being referred to MASH.

Across the three event descriptions, Suspects were on average aged 33 to 38 , with current research also concluding that perpetrators of harm or abuse are typically aged 30 (Osadan and Reid, 2015; Hester and Westmarland, 2006). Referrals to MASH therefore, were influenced by the age of the LR and Suspect, reiterating the belief that whilst everyone is potentially vulnerable, some age groups are more likely to be referred to MASH as a victim or Suspect.

The reason to why a referral to MASH was made, alongside the relationship between Suspect and LR, also differed across the event descriptions. VC referrals primarily cited Sexual Abuse as a referral reason, with Parent being identified as the main relationship status. Previous research has also found sexual abuse to be a prominent form of abuse in childhood, with parents, or acquaintances, often being the source of such abuse (Townsend, 2013; Joubert et al., 2012; Amos et al., 2011).

Most VA referrals cited a referral reason of Physical Abuse, with previous research also concluding that adults are usually referred to safeguarding boards due to neglect or physical abuse (HSCIC, 2015; Trainor and Penhale, 2015). The relationship between Suspect and LR in VA cases was predominantly stated to be Other. Whilst this relationship status was 
RUNNING HEAD: Investigating the Characteristics of Vulnerable Referrals made to a MultiAgency Safeguarding Hub (MASH).

unclear, it can be deduced that the Suspect and LR were known to one another, since there was an option of 'Stranger'. However, the Suspect and LR were not family members, nor was the Suspect in a position of trust, since these were also presented as separate options. This, supports the assertion that vulnerable adults are generally harmed or abused by someone they know (HSCIC, 2014; Fyson and Kitson, 2012; O'Keeffe et al., 2007).

DA cases principally involved Partners or Ex-Partners, with domestic abuse being the main referral reason. Once again, this finding reiterates existing understandings of domestic abuse, particularly the notion that domestic abuse occurs between two people who are, or have been, in a relationship (Office for National Statistics, 2014; Reijnders, 2014; Johnson and Ferraro, 2000). Therefore, the relationship status between Suspect and LR influenced referral rates to $\mathrm{MASH}$, with a vulnerable person most likely to be harmed or abused by someone they knew.

In terms of management, the results illustrated that referrals to this MASH site occurred within 7-days of a police officer attending a risky situation, supporting the notion that MASH has enabled vulnerability to be identified early. However, the demand placed upon MASH may prevent it from managing vulnerability at the earliest opportunity. At this MASH site, the time needed to process a referral differed by the level of risk assigned to a case, alongside the event description linked to a case. For instance, the protocol for high-risk cases was that they should be processed within 4-hours of being referred, whilst standardrisk referrals have a processing time of 72-hours. However, the average time taken to process a referral was 10 days, regardless of risk level assigned, supporting the notion that the demand placed upon MASH may outweigh the availability of resources. The processing times linked to VC referrals exemplify this concern, since such cases were generally assigned 
RUNNING HEAD: Investigating the Characteristics of Vulnerable Referrals made to a MultiAgency Safeguarding Hub (MASH).

a higher risk level than VA or DA referrals. This suggests that practitioners perceived cases involving children as more urgent than those relating to adults, possibly because of the age of children and their dependency on others to grow and develop (Siegel, 2015). Despite this recognition, few VC referrals were processed by MASH within a 24-hour period, with the absence of immediate safeguarding interventions potentially increasing the likelihood of children being exposed to further harm or abuse (Solomon and Asberg, 2012). The complexity of a case may have impacted upon the length of time needed to process a case, with immediate safeguarding interventions possibly being applied prior to the referral being formal closed. Therefore, whilst the quantitative data questions MASHs capacity to effectively respond to risk at the earliest opportunity, a better understanding of decisionmaking processes within MASH may help to explain why processing times are lengthy and exceed predefined timeframes.

\section{Limitations and Future Considerations.}

Data used throughout this study was provided by the police force associated with the MASH site, since information relating to all MASH referrals is stored on their databases. This reliance on police data proved problematic, since recording practices were inconsistent, with those responsible for submitting a referral, namely police officers, not always accurately reporting case characteristics. For instance, the research found that age, gender and ethnicity of LR and Suspects, alongside relationship status, was either incorrectly recorded or not recorded at all. Equally, the findings of this report primarily relate to just one MASH location, potentially reducing the extent to which findings can be generalised to other MASH locations. 
RUNNING HEAD: Investigating the Characteristics of Vulnerable Referrals made to a MultiAgency Safeguarding Hub (MASH).

Due this study only focusing upon one MASH location in the North West of England, characteristics associated with LR and Suspects, alongside referral and processing times, may not be representative of other MASH locations. To increase the validity and generalisability of findings therefore, a similar study needs to be conducted across other MASH sites. If this is achieved, the likelihood of reliably identifying risk factors associated with vulnerability, at a national level, would increase.

Equally, this study has only focused upon the data recorded on a MASH referral form, overlooking the way in which MASH practitioners use such information to process referrals. Thus, to better understand the way in which MASH identifies, manages and safeguards vulnerable individuals, the operational workings of MASH needs to be investigated. To achieve this aim, a practitioner perspective of MASH is required, with specific attention given to the way in which practitioners have embedded practices and processes into the MASH framework.

\section{Conclusion.}

Since 2010, Multi-Agency Safeguarding Hubs (MASH) have started to feature within safeguarding practices, however, the purpose of MASH and who is referred into them has often been overlooked. To bridge this gap in knowledge, this study examined the referrals to a MASH in the North of England. It was found that whilst MASH protects a variety of vulnerable individuals, referrals fell into one of three groups: Vulnerable Children (VC); Vulnerable Adults (VA); and Domestic Abuse (DA). Within these groups, VC referrals were most likely to be referred as medium-risk, involve LR aged 10 years old, with Sexual Abuse being the primary referral reason. VA cases were predominantly referred as standard-risk 
RUNNING HEAD: Investigating the Characteristics of Vulnerable Referrals made to a MultiAgency Safeguarding Hub (MASH).

cases, with LR being older, on average, than Suspects. Likewise, DA referrals were mainly referred as standard-risk cases, with Partner or Ex-partner being the most cited relationship status between Suspect and LR. In terms of processing times, MASH processed DA cases much quicker than VC and VA referrals. Thus, whilst no two referrals to MASH were the same, referral rates to MASH were influenced by an individual's gender, age and ethnicity, as well as the relationship between the LR and Suspect. Furthermore, the study has illustrated that whilst MASH aims to process all referrals within a 72-hour timeframe, the average processing time exceeds this maximum timeframe, raising questions around MASHs ability to manage risk at the earliest opportunity. 
RUNNING HEAD: Investigating the Characteristics of Vulnerable Referrals made to a MultiAgency Safeguarding Hub (MASH).

\section{REFERENCES.}

Abramsky, T., Watts, C.H., Garcia-Moreno, C., Devries, K., Kiss, L., Ellsberg, M., Jansen, H.A. and Heise, L. 2011, "What factors are associated with recent intimate partner violence? Findings from the WHO multi-country study on women's health and domestic violence", BMC public health, vol. 11, pp. 109-2458-11-109.

Allen, C. and Wilde, B. 2013, Birmingham Multi Agency Safeguarding Hub (MASH) Review, March 2013, Birmingham City Council, Birmingham.

Amos, J., Furber, G. and Segal, L. 2011, "Understanding maltreating mothers: a synthesis of relational trauma, attachment disorganization, structural dissociation of the personality, and experiential avoidance", Journal of Trauma and Dissociation, vol. 12, no. 5, pp. 495-509.

Braams, B. R., van Duijvenvoorde, A. C., Peper, J. S., \& Crone, E. A. 2015, “Longitudinal changes in adolescent risk-taking: a comprehensive study of neural responses to rewards, pubertal development, and risk-taking behaviour", Journal of Neuroscience, vol. 35 , no. 18 , pp. $7226-7238$.

Boulton, N., Ladbury, B., and King, J. 2013, Sharing Information in the MASH. Available: http://www.londonscb.gov.uk/mash/ (accessed 15 December 2016).

Caldicott, F. 1997, Report on the Review of Patient-Identifiable Information, Department of Health, London. 
RUNNING HEAD: Investigating the Characteristics of Vulnerable Referrals made to a MultiAgency Safeguarding Hub (MASH).

Cooper, L. 2018, How to recognise and respond to potential child abuse and neglect, The Pharmaceutical Journal, vol. 300, no. 7910, [Online]. Available: https://www.pharmaceutical-journal.com/learning/how-to-recognise-and-respond-topotential-child-abuse-and-neglect/20204393.article (accessed 20 April 2018).

Crockett, R., Gilchrist, G., Davies, J., Henshall, A., Hoggart, L., Chandler, V., Sims, D. and Webb, J. 2013, "Assessing the early impact of Multi Agency Safeguarding Hubs (MASH) in London" (Online). Available: http://www.londonscb.gov.uk/wpcontent/uploads/2016/04/mash_report_final.pdf (accessed 11 September 2015).

Cullinan, M. July 2013, Lancashire Improving Futures Programme.

Available: www.lancashirechildrenstrust.org.uk/web/viewdoc.asp?id=103050 (18/06/2015).

Department for Education, 2015, Characteristics of children in need: 2014 to 2015. Available: https://assets.publishing.service.gov.uk/government/uploads/system/uploads/attachm ent_data/file/469737/SFR41-2015_Text.pdf (19/04/2018).

Finkelhor, D., Vanderminden, J., Turner, H., Hamby, S. and Shattuck, A. 2014, "Child maltreatment rates assessed in a national household survey of caregivers and youth", Child abuse and neglect, vol. 38, no. 9, pp. 1421-1435.

Furedi, F. 2013, Moral crusades in an age of mistrust: The Jimmy Savile scandal, Palgrave Macmillan. 
RUNNING HEAD: Investigating the Characteristics of Vulnerable Referrals made to a MultiAgency Safeguarding Hub (MASH).

Fyson, R. and Kitson, D. 2012, "Outcomes following adult safeguarding alerts: a critical analysis of key factors", The Journal of Adult Protection, vol. 14, no. 2, pp. 93-103.

Gloucestershire Multi Agency Safeguarding Hub (MASH), 2014. Frequently asked questions for professionals. Available: www.gloucestershire.gov.uk/gsab/CHttpHandler.ashx?id=5797 3andp=0 (accessed 2 May 2014).

Golden, S., Aston, H., Durbin, B. and Local Government Association 2011, Devon MultiAgency Safeguarding Hub: Case-Study Report, NFER.

Gullone, E., \& Moore, S. 2000. “Adolescent risk-taking and the five-factor model of personality," Journal of Adolescence, vol. 23, no. 4, pp. 393-407.

Hanson, J., Carwardine, J., Chapman, P., Ross, D. and Massey, K. and Bamford, N. 2015, Safeguarding Annual Report: 2014 - 2015, East Lancashire CCGs, Preston.

Haringey, L. 2009, "Serious case review: baby Peter", Haringey, Local Safeguarding Children Board.

Hassan, M. A., Gary, F., Killion, C., Lewin, L. and Totten, V. 2015, "Patterns of Sexual Abuse among Children: Victims' and Perpetrators' Characteristics", Journal of Aggression, Maltreatment and Trauma, vol. 24, no. 4, pp. 400-418.

Health and Social Care Information Centre (HSCIC) 2014, Abuse of Vulnerable Adults in England: 2012-13, Final Report, Experimental Statistics, Health and Social Care Information Centre, London. 
RUNNING HEAD: Investigating the Characteristics of Vulnerable Referrals made to a MultiAgency Safeguarding Hub (MASH).

Health and Social Care Information Centre (HSCIC) 2015, Safeguarding Adults Annual Report, England 2014-15 Experimental Statistics, Health and Social Care Information Centre, London.

Hester, M. and Westmarland, N. 2006, Service Provision for Perpetrators of Domestic Violence, Bristol: University of Bristol.

HM Government 2015, Working Together to Safeguard Children, Department of Education, London.

Home Office July 2014, Multi Agency Working and Information Sharing Project: Final report. Available: https://www.gov.uk/government/uploads/system/uploads/attachment_data /file/338875/MASH.pdf (accessed 18 October 2016).

Johnson, M.P. and Ferraro, K.J. 2000, "Research on domestic violence in the 1990s: Making distinctions", Journal of Marriage and Family, vol. 62, no. 4, pp. 948-963.

Joubert, D., Webster, L. and Hackett, R.K. 2012, "Unresolved attachment status and traumarelated symptomatology in maltreated adolescents: An examination of cognitive mediators", Child Psychiatry and Human Development, vol. 43, no. 3, pp. 471-483.

Laming, L. 2009, "The Protection of Children in England: A Progress Report 2009", London, The Stationery Office.

Laming, W. 2003, "The Victoria Climbié Inquiry-Report of an Inquiry by Lord Laming Presented to Parliament by the Secretary of State for Health and the Secretary of state for the Home Department by Command of Her Majesty", London: The Stationery Office. 
RUNNING HEAD: Investigating the Characteristics of Vulnerable Referrals made to a MultiAgency Safeguarding Hub (MASH).

Lancashire CYP Trust Board and LSCB 2012, Transforming Service Delivery Through MASH and Multi-Agency Delivery Hubs.

Available: http://www.lancashirechildrenstrust.org.uk/calendar/views/entries/showAtt .asp?id=21077 (accessed 2 April 2015).

Livingstone, S., and Palmer, T. (2012) Identifying vulnerable children online and what strategies can help them, UK Safer Internet Centre, London, UK.

London Safeguarding Children Board 2013, London MASH Information Sharing Guidance. Available: http://www.londonscb.gov.uk/mash/ (accessed 30 September 2015).

Modleski, T. 2015, The women who knew too much: Hitchcock and feminist theory, Routledge.

NHS Choices 2016, Stages of puberty: what happens to boys and girls. Available: https://www.nhs.uk/livewell/puberty/pages/puberty-signs.aspx (accessed 20 April 2018).

Nib Consulting 2014, Multi Agency Safeguarding Hubs. Available: http://www.nibconsulting.co.uk/mash.html (accessed 01 December 2017).

Norfolk Adult Safeguarding Board 2014, Norfolk Multi-Agency Safeguarding Adults Procedures, Norfolk County Council, Norfolk.

NSPCC 2018, Online Abuse: Who is Affected. Available: https://www.nspcc.org.uk/preventing-abuse/child-abuse-and-neglect/onlineabuse/who-is-affected/ (accessed 20 April 2018). 
RUNNING HEAD: Investigating the Characteristics of Vulnerable Referrals made to a MultiAgency Safeguarding Hub (MASH).

Ofcom 2017, Children and Parents: Media Use and Attitudes Report. Available:

https://www.ofcom.org.uk/_data/assets/pdf_file/0020/108182/children-parentsmedia-use-attitudes-2017.pdf (accessed 22 April 2018).

Office for National Statistics 2014, Chapter 4 - Intimate Personal Violence and Partner Abuse. Available: http://www.ons.gov.uk/ons/dcp171776_352362.pdf (accessed 05 May 2015).

Office for National Statistics 2016, Abuse during childhood: Findings from the Crime Survey for England and Wales, year ending March 2016. Available: https://www.ons.gov.uk/peoplepopulationandcommunity/crimeandjustice/articles/ab useduringchildhood/findingsfromtheyearendingmarch2016crimesurveyforenglandand wales (accessed 20 March 2018).

Office for National Statistics 2017, Domestic abuse in England and Wales: year ending March 2017. Available:

https://www.ons.gov.uk/peoplepopulationandcommunity/crimeandjustice/bulletins/d omesticabuseinenglandandwales/yearendingmarch2017 (accessed 20 March 2018).

O'Keeffe, M., Britain, G. and Relief, C. 2007, UK study of abuse and neglect of older people: Prevalence survey report, Comic Relief.

Osadan, R. and Reid, E. 2015, "Child Sexual Abuse and Sexual Aggression in Children", International Journal of Humanities and Social Science, vol. 5, no. 6(1), pp. 31-42. 
RUNNING HEAD: Investigating the Characteristics of Vulnerable Referrals made to a MultiAgency Safeguarding Hub (MASH).

Radford, L., Corral, S., Bradley, C., Fisher, H., Bassett, C., Howat, N. and Collishaw, S. 2011, Child Abuse and Neglect in the UK Today, NSPCC, London.

Runyan, D., Wattam, C., Ikeda, R., Hassan, F., \& Ramiro, L. 2002, "Child abuse and neglect by parents and other caregivers" in World Report on Violence and Health, ed. Krug, E. G., Dahlberg, L., Mercy, J. A., Zwi, A. B., and Lozano, R., World Health Organization, Geneva, pp. 57-86.

SafeLives 2015, Insights IDVA National Dataset 2013-14., SafeLives, Bristol.

Siegel, D. J. 2015, The developing mind: How relationships and the brain interact to shape who we are. London: Guilford Publications.

Solomon, D., \& Asberg, K. 2012, “Effectiveness of child protective services interventions as indicated by rates of recidivism", Children and Youth Services Review, vol. 34, no. 12, pp 2311-2318.

Stevens, M., Norrie, C., Manthorpe, J., Hussein, S., Moriarty, J. and Graham, K. 2016, "Models of adult safeguarding in England: Findings from a study of costs and referral outcomes", British Journal of Social Work, pp. bcw025.

Townsend, C. 2013, Prevalence and consequences of child sexual abuse compared with other childhood experiences. Charleston: Darkness to Light.

Trainor, P. and Penhale, B. 2015, "A review of factors which potentially influence decisions in adult safeguarding investigations", The Journal of Adult Protection, vol. 17, no. 1. 


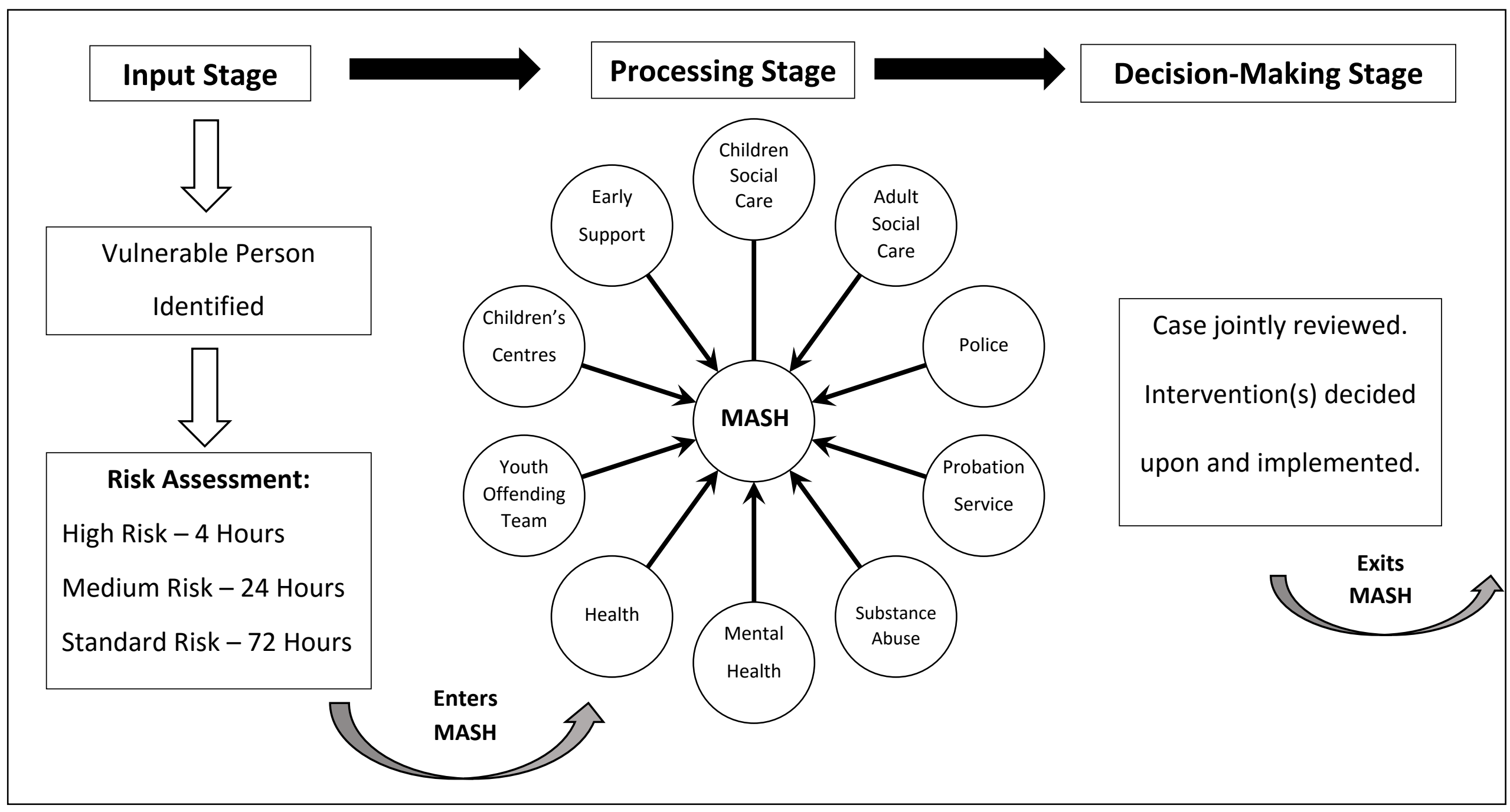

Figure 1: Example of MASH Process. 
RUNNING HEAD: Investigating the Characteristics of Vulnerable Referrals made to a MultiAgency Safeguarding Hub (MASH).

\section{Recorded Date:}

Police attend and incident and identify a vulnerable person.

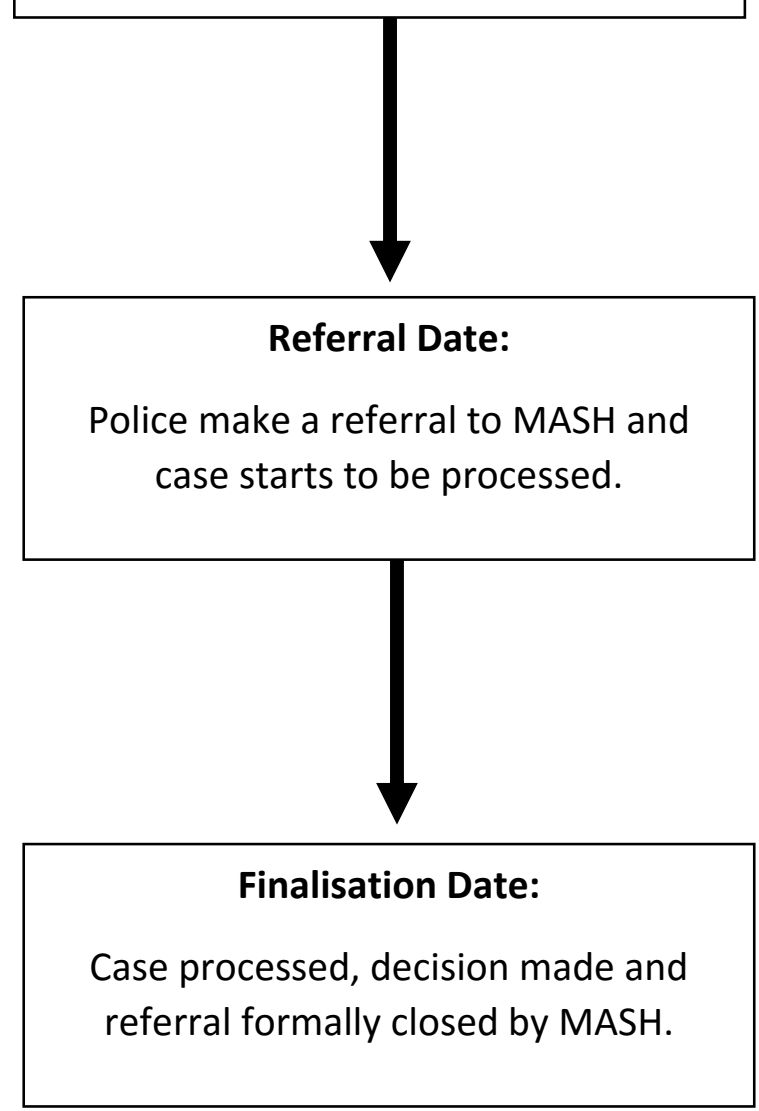

Figure 2: Timeline of Key Dates Recorded by MASH. 
Table 1: Overview of Demand by Event Description.

\begin{tabular}{|c|c|c|c|c|c|c|c|c|c|c|c|c|c|c|c|c|}
\hline \multirow{3}{*}{ Factor } & \multicolumn{16}{|c|}{ Event Description } \\
\hline & \multicolumn{4}{|c|}{$\begin{array}{c}\text { VC } \\
(N=10,691)\end{array}$} & \multicolumn{4}{|c|}{$\begin{array}{c}\text { VA } \\
(N=10,342)\end{array}$} & \multicolumn{4}{|c|}{$\begin{array}{c}\text { DA } \\
(N=30,231)\end{array}$} & \multicolumn{4}{|c|}{$\begin{array}{l}\text { All Referrals } \\
(n=51,264)\end{array}$} \\
\hline & \multicolumn{2}{|c|}{ LR } & \multicolumn{2}{|c|}{ Suspect } & \multicolumn{2}{|c|}{ LR } & \multicolumn{2}{|c|}{ Suspect } & \multicolumn{2}{|c|}{ LR } & \multicolumn{2}{|c|}{ Suspect } & \multicolumn{2}{|c|}{ LR } & \multicolumn{2}{|c|}{ Suspect } \\
\hline Risk Level* & \multicolumn{2}{|c|}{$N=10,691$} & \multicolumn{2}{|c|}{$N=11,451$} & \multicolumn{2}{|c|}{$N=10,342$} & \multicolumn{2}{|c|}{$N=10,603$} & \multicolumn{2}{|c|}{$N=30,231$} & \multicolumn{2}{|c|}{$N=30,667$} & \multicolumn{2}{|c|}{$N=51,264$} & \multicolumn{2}{|c|}{$N=52,721$} \\
\hline High & 3,462 & $32 \%$ & 3,833 & $33 \%$ & 1,500 & $15 \%$ & 1,565 & $15 \%$ & 2,991 & $10 \%$ & 3,062 & $10 \%$ & 7,953 & $16 \%$ & 8,460 & $16 \%$ \\
\hline Medium & 5,083 & $48 \%$ & 5,383 & $47 \%$ & 4,321 & $42 \%$ & 4,438 & $42 \%$ & 9,173 & $30 \%$ & 9,312 & $30 \%$ & 18,577 & $36 \%$ & 19,133 & $36 \%$ \\
\hline Standard & 2,146 & $20 \%$ & 2,235 & $20 \%$ & 4,521 & $44 \%$ & 4,600 & $43 \%$ & 18,067 & $60 \%$ & 18,293 & $60 \%$ & 24,734 & $48 \%$ & 25,128 & $48 \%$ \\
\hline Gender* & \multicolumn{2}{|c|}{$N=10,067$} & \multicolumn{2}{|c|}{$N=5,095$} & \multicolumn{2}{|c|}{$N=9,971$} & \multicolumn{2}{|c|}{$N=1,962$} & $N=29$ & 525 & $N=29$ & 521 & $N=49$ & 563 & $N=36$ & 578 \\
\hline Female & 5,547 & $55 \%$ & 1,855 & $36 \%$ & 5,260 & $53 \%$ & 490 & $25 \%$ & 22,762 & $77 \%$ & 5,603 & $19 \%$ & 33,569 & $68 \%$ & 7,948 & $22 \%$ \\
\hline Male & 4,520 & $45 \%$ & 3,240 & $64 \%$ & 4,711 & $47 \%$ & 1,472 & $75 \%$ & 6,763 & $23 \%$ & 23,918 & $81 \%$ & 15,994 & $32 \%$ & 28,630 & $78 \%$ \\
\hline Age* & $N=10$ & 591 & $N=5$, & 120 & $N=1$ & & $N=1$ & 34 & $N=30$ & 231 & $N=30$ & 191 & $N=51$ & 264 & $N=37$ & 245 \\
\hline Mean & 10 (SD & $5.32)$ & 33 (SD & $=12.7)$ & 49 (SD & $.87)$ & 38 (SD & $6.25)$ & $35(S D=$ & 2.79) & $34(S D=$ & 11.81) & $33(S D=$ & $9.13)$ & $34(S D=$ & 12.26) \\
\hline Ethnicity & $N=9$ & & $N=4$ & 841 & $N=1$ & & $N=1$ & 49 & $N=29$ & 720 & $N=29$ & 677 & $N=49$ & 564 & $N=36$ & 367 \\
\hline Asian & $592^{*}$ & $6 \%$ & 371 & $8 \%$ & $496 *$ & $5 \%$ & 160 & $9 \%$ & $1,796^{*}$ & $6 \%$ & 2,137 & $7 \%$ & $2,884^{*}$ & $6 \%$ & 2,668 & $7 \%$ \\
\hline Black & $100 *$ & $1 \%$ & 64 & $1 \%$ & $106^{*}$ & $1 \%$ & 37 & $2 \%$ & $251 *$ & $1 \%$ & 411 & $1 \%$ & $457^{*}$ & $1 \%$ & 512 & $1 \%$ \\
\hline White & $9,006^{*}$ & $92 \%$ & 4,387 & $91 \%$ & $9,488^{*}$ & $94 \%$ & 1,644 & $89 \%$ & $27,559 *$ & $93 \%$ & 27,001 & $91 \%$ & $46,053^{*}$ & $93 \%$ & 33,032 & $91 \%$ \\
\hline Other & $37^{*}$ & $<1 \%$ & 19 & $<1 \%$ & $19 *$ & $<1 \%$ & 8 & $<1 \%$ & $114^{*}$ & $<1 \%$ & 128 & $<1 \%$ & 170* & $<1 \%$ & 15 & $<1 \%$ \\
\hline Referral Reason & $N=10$ & 591 & & & $N=1$ & & & & $N=30$ & 231 & & & $N=51$ & 264 & & \\
\hline Channel* & 35 & $<1 \%$ & - & - & 39 & $<1 \%$ & - & - & 4 & $<1 \%$ & - & - & 78 & $<1 \%$ & - & - \\
\hline CSE* & 1,310 & $12 \%$ & - & - & 80 & $1 \%$ & - & - & 15 & $<1 \%$ & - & - & 1,405 & $3 \%$ & - & - \\
\hline Domestic Abuse* & 550 & $5 \%$ & - & - & 379 & $4 \%$ & - & - & 30,231 & $100 \%$ & - & - & 31,160 & $61 \%$ & - & - \\
\hline Forced Marriage* & 21 & $<1 \%$ & - & - & 42 & $<1 \%$ & - & - & 20 & $<1 \%$ & - & - & 83 & $<1 \%$ & - & - \\
\hline $\mathrm{HBA}^{*}$ & 37 & $<1 \%$ & - & - & 99 & $1 \%$ & - & - & 124 & $<1 \%$ & - & - & 260 & $1 \%$ & - & - \\
\hline $\mathrm{MFH}^{*}$ & 778 & $7 \%$ & - & - & 634 & $6 \%$ & - & - & 64 & $<1 \%$ & - & - & 1,476 & $3 \%$ & - & - \\
\hline Neglect* & 1,945 & $18 \%$ & - & - & 818 & $8 \%$ & - & - & 119 & $<1 \%$ & - & - & 2,882 & $6 \%$ & - & - \\
\hline Other* & 49 & $<1 \%$ & - & - & 123 & $1 \%$ & - & - & 26 & $<1 \%$ & - & - & 198 & $<1 \%$ & - & - \\
\hline Physical Abuse* & 1,799 & $17 \%$ & - & - & 1,253 & $12 \%$ & - & - & 1,977 & $7 \%$ & - & - & 5,029 & $10 \%$ & - & - \\
\hline Sexual Abuse* & 2,106 & $20 \%$ & - & - & 809 & $8 \%$ & - & - & 226 & $1 \%$ & - & - & 3,141 & $6 \%$ & - & - \\
\hline Relationship* & & Pare & & & & Oth & & & & Partn & r/Ex & & & Partn & $\mathrm{r} / \mathrm{Ex}$ & \\
\hline
\end{tabular}


RUNNING HEAD: Investigating the Characteristics of Vulnerable Referrals made to a MultiAgency Safeguarding Hub (MASH).

Table 2: Average Referral and Processing Times by Event Description and Risk Level.

\begin{tabular}{|c|c|c|}
\hline \multirow[b]{2}{*}{ Factor } & \multicolumn{2}{|c|}{ Timeframe (Mean) } \\
\hline & $\begin{array}{l}\text { Referral Time } \\
\qquad(n=51,264)\end{array}$ & $\begin{array}{l}\text { Processing Time } \\
(n=51,264)\end{array}$ \\
\hline \multicolumn{3}{|l|}{ Event Description } \\
\hline VC & 6 Days (SD=64.27)* & 14 Days $(S D=64.73) *$ \\
\hline VA & 6 Days $(S D=59.36) *$ & 13 Days (SD=59.70)* \\
\hline DA & 1 Day (SD=19.16)* & 8 Days $(S D=20.35) *$ \\
\hline All Referrals & 3 Days $(S D=41.77)^{*}$ & 10 Days $(S D=42.64) *$ \\
\hline \multicolumn{3}{|l|}{ Risk Level } \\
\hline High & 6 Days $(S D=73.60)^{*}$ & 10 Days $(S D=73.96) *$ \\
\hline Medium & 3 Days (SD=34.11)* & 13 Days (SD=34.94)* \\
\hline Standard & 3 Days $(S D=33.16)^{*}$ & 8 Days (SD=33.75)* \\
\hline
\end{tabular}

